\title{
O Papel das Redes e da Capacidade de Conversão de Conhecimento no Desenvolvimento de Spin-Offs Acadêmicas
}

The Role of Networks and Knowledge Conversion Capacity in the Development of Academic Spin-Offs

\author{
Brenno Buarque ${ }^{1}$ \\ Ana Cristina Batista dos Santos ${ }^{2}$ \\ Naiderson Ferreira de Lucena ${ }^{3}$ \\ Rafaela Cajado Magalhães ${ }^{4}$ \\ Herus Orsano Machado ${ }^{5}$
}

\section{Resumo}

A presente pesquisa tem como objetivo compreender o papel das redes e da capacidade de conversão de conhecimento no desenvolvimento de spin-offs acadêmicas. A pesquisa de campo processou-se por entrevistas detalhadas, realizadas com cinco gestores e sócios de diferentes spin-offs na área de biotecnologia, sendo orientadas por um roteiro semiestruturado. Utilizou-se também a técnica de análise de conteúdo para análise das entrevistas. Considerando os resultados dessa pesquisa, foi possível perceber que as redes das spin-offs fomentam a formação de parcerias, as quais viabilizam a construção de know-how, e, consequentemente, o aprendizado e a capacidade de conversão de conhecimento, o que leva ao desenvolvimento das instituições estudadas.

Palavras-chave: Spin-offs acadêmicas; Redes de cooperação; Capacidade de conversão de conhecimento; Transferência de tecnologia; Empreendedorismo acadêmico.

\begin{abstract}
The paper presents results of research that aimed to understand the role of networks and the ability to convert knowledge in the development of academic spin-offs. The data collection consisted of detailed interviews with five managers and partners from different spin-offs. The technique used to analyze the interviews was thematic content analysis. As outstanding results, it was possible to identify that the spin-off networks foster the formation of partnerships, which enable the construction of know-how, and, consequently, the learning and the ability to convert knowledge, which leads to the development of the studied institutions.
\end{abstract}

Keywords: Academic spin-offs; Cooperation networks; Knowledge conversion capability; Technology transfer; Academic entrepreneurship. 


\section{INTRODUÇÃO}

As universidades passaram por revoluções acadêmicas e novas funções Ihes foram atribuídas. A última, ocorrida no final do século XX, atribuiu-lhe a função de comercializar o conhecimento que produz, o que Etzkowitz (1998, p. 826) define como "capitalização do conhecimento" (Frota \& Freitas, 2018). Assim, Etzkowitz (1998) afirma que entre as décadas de 1980 e 1990 foi crescente o número de cientistas que passaram a criar empresas e agiram nesta direção de levar o conhecimento produzido para os mercados.

Nesse contexto, nas últimas décadas, as universidades de ponta não ficaram limitadas à produção e à transferência de conhecimento, mas também adotaram uma nova função, a de transferência tecnológica, que reitera o papel da universidade como um agente socioeconômico (Etzkowitz, 1998). Neste cenário, são as spin-offs acadêmicas - empresas criadas dentro de instituições de ensino para a comercialização de pesquisas e conhecimentos -, que assumem um dos mais relevantes papéis na transferência de tecnologia.

Desta forma, o papel de transferência de tecnologia realizado pelas spin-offs acadêmicas acontece a partir do trabalho de pesquisadores, estudantes e profissionais que passam a atuar no campo do empreendedorismo (Etzkowitz, 1998; Perez \& Sánchez, 2003), levando suas pesquisas e conhecimentos para o setor produtivo, possibilitando a comercialização de tecnologias desenvolvidas no ambiente acadêmico ou nas organizações empresariais.

Etzkowitz (1998) afirma que nos mais avançados segmentos do sistema universitário mundial, as universidades passaram a desempenhar uma função de agente promotor do desenvolvimento econômico, para além das funções tradicionais de ensino e pesquisa, gerando mudanças na forma como os pesquisadores tratam seu papel na universidade.

O fenômeno do surgimento e atuação das spin-offs acadêmicas têm sido estudado em seus diversos aspectos, desde seus elementos internos que facilitaram suas performances, como o perfil da equipe fundadora (Walter, Auer, \& Ritter, 2006; Soetanto \& Van Geenhuizen, 2015), até complexos aspectos externos como a relação entre a capacidade de financiamento de spin-offs e as redes em que estão inseridas (Huynh et al., 2017). No entanto, são escassos os estudos que abordam a influência das redes de relacionamento na capacidade de conversão de conhecimento e no desenvolvimento dessas empresas.

Apesar da literatura enfatizar a importância das redes de relacionamento para uma spin-off acadêmica, principalmente no que diz respeito à transferência de tecnologia e ao seu desempenho (Perez \& Sánchez, 2003; Walter, Auer, \& Ritter, 2006; Soetanto \&Van Geenhuizen, 2015; Huynh et al., 2017), há poucos estudos que abordem a relação entre as redes, a capacidade de conversão de conhecimento e o desenvolvimento dessas empresas.

Faltam pesquisas que procurem compreender como as redes das spin-offs influenciam no know-how (conhecimento) técnico e comercial dos membros, isto é, sua capacidade de conversão de conhecimento, que impacta no desenvolvimento tecnológico dos produtos nessas empresas.

Assim, é importante explorar esta lacuna da literatura, de forma a compreender como as spin-offs são influenciadas pelas redes nas quais estão inseridas, bem como sobre a importância destas redes na capacidade de transformar o conhecimento em produto comercializável - representado neste estudo pela capacidade de conversão de conhecimento de produto.

O grande desafio da spin-off acadêmica é tornar comercializável a tecnologia e o conhecimento técnicocientífico que seus membros possuem, buscando, com os resultados desta pesquisa, contribuir com a literatura ao elencar como as redes contribuem para a superação deste desafio. Deste modo, este trabalho parte da seguinte questão de pesquisa: de que forma a inserção em redes de relacionamento influencia na capacidade de conversão de conhecimento de produto e no desenvolvimento de spin-offs acadêmicas?

Os relacionamentos que essas empresas desenvolvem influenciam também no aprendizado na área de gestão e de transferência tecnológica. Esses aspectos impactam no desenvolvimento dessas empresas, o que torna pertinente pesquisas que envolvam tais temas nos estudos da área. Assim, a pesquisa é orientada pelo objetivo de compreender o papel das redes e da capacidade de conversão de conhecimento no desenvolvimento de spin-offs acadêmicas (empresas que cormecializam conhecimentos).

\section{REVISÃO DE LITERATURA}

\subsection{Spin-Offs Acadêmicas e Redes de Cooperação}

Soetanto e Van Geenhuizen (2015) estudam a importância das redes universitárias para a atração de financiamento para as spin-offs acadêmicas. Os autores evidenciam que o fato das spin- offs participarem de redes de relacionamento dentro da universidade possui relação direta com a sua capacidade de obtenção de financiamento, e que tal capacidade se amplia quando as spin-offs desenvolvem contato com redes não universitárias.

A associação de redes de relacionamento em spin-offs acadêmicas é especialmente importante por estar relacionada com a comercialização de um novo conhecimento em um setor nos quais os participantes não possuem experiência de mercado (Soetanto \& Van Geenhuizen, 2015; Huynh et al., 2017). A associação em redes, envolvendo 
a universidade e especialistas na área, é importante principalmente nos primeiros anos, fase em que a spin-off necessita de know-how para desenvolvimento de produto e transferência de tecnologia.

A literatura científica internacional sobre redes contribui com importantes avanços nos últimos 20 anos, onde o foco de abordagem temática dos pesquisadores internacionais refere-se aos modelos teóricos relacionados às redes de cooperação, redes sociais, governança e redes de políticas públicas (Freitas et al., 2017).

No que concerne as redes de cooperação científica, este trabalho adota a visão de Hilário e Grácio (2018) que defendem a formação dessas redes, de forma espontânea e auto organizada para produzir conhecimento, ou seja, considerando que as redes de cooperação científica são uma realidade do ambiente acadêmico.

Sabe-se, ainda, que estas têm despertado a atenção de pesquisadores nacionais e internacionais, visto que, os níveis de organização dos atores dessas redes têm aumentado a cooperação em diferentes áreas de conhecimento científico e tecnológico, incluindo as produções técnicas patenteáveis e o processo de criação de spin-offs (Hilário \& Grácio, 2018).

No contexto organizacional, a atuação nessas redes promove vantagens competitivas em um ambiente de estrutura dinâmica, onde as ações dos atores são de característica uniforme, porém sem uma ordem de comando central, o que propicia uma maior adaptabilidade organizacional, gerando altos ganhos à rede (Thompson, 2003).

Na perspectiva da construção de conhecimento científico, diversos autores indicam as formas de organização das redes de cooperação a fim de proporcionar compartilhamento de conhecimentos e informações no desenvolvimento e promoção da inovação (Breschi \& Malerba, 2005; Balestrin, Verschoore, \& Reyes Junior, 2010; Funk, 2014).

No caso das redes de spin-offs acadêmicas, seus gestores geralmente decidem participar das mesmas visando ao objetivo comum de compartilhar conhecimento gerador de inovação, aumentando, consequentemente, a capacidade de inovação dos membros (Breschi \& Malerba, 2005; Funk, 2014).

Além disso, os gestores de spin-offs apresentam grande interesse em adquirir know-how para possível transferência tecnológica de seus produtos. A associação em redes de cunho tecnológicose torna, assim, uma alternativa para que tal transferência se efetive. Tsai (2001) comprova que o nível de engajamento de uma empresa em uma rede interorganizacional está relacionado tanto com a inovação quanto com a performance nos negócios, através da transferência de conhecimento entre as mesmas e a capacidade absortiva de cada uma delas.

Brass et al. (2004) afirmam que a rede de cooperação interorganizacional compreende três níveis de composição: 1) os integrantes dos grupos, 2) os grupos inseridos nas associações e 3) as associações que compõem a rede. No caso das spin-offs acadêmicas, os membros possuem interesses de desenvolvimento de produtos, transferência de tecnologia, compartilhamento de propriedade intelectual, além de aprendizado em mecanismos de gestão, que impactam na relação dos grupos inseridos em organizações e nas organizações que compõem as redes de pesquisa e conhecimento, algo que, normalmente, não representa a realidade de conhecimento e atuação de pesquisadores (Soetanto \& Van Geenhuizen, 2015; Huynh et al., 2017).

Huynh et. al. (2017) estudam as capacidades e as redes dos membros fundadores de uma spin-off durante a fase de criação. Em seus resultados, mostram que os membros fundadores aumentam sua capacidade empreendedora através de suas redes, com impacto na fase de crescimento dessas empresas. Os autores enfatizam a necessidade das spin-offs se associarem às redes para obtenção de vantagens, principalmente, no que concerne à transferência de tecnologia, destacando a importância dos escritórios responsáveis por essa ação, que possibilitam o envolvimento das spin- offs com participantes de redes possuidores do potencial alavancador de desempenho.

Outro fator importante para a associação de redes é o conceito de network capability. Walter, Auer e Ritter (2006) definem network capability como a habilidade para iniciar, manter e utilizar-se de relacionamentos com diversos parceiros externos.

Em sua pesquisa, os autores demonstram que a network capability está relacionada com o desempenho das spin-offs acadêmicas, ao apontarem seis critérios de desempenho que são influenciados pela network capability: i) crescimento de vendas; ii) vendas por funcionário; iii) realização de lucro; iv) percepção da qualidade do relacionamento com o cliente; v) vantagens competitivas percebidas; e vi) sobrevivência a longo prazo.

No ensejo desta pesquisa, a temátida de redes foi abordada com foco no conceito de redes de cooperação interorganizacionais, tendo como base a corrente teórica da Abordagem de Estratégias Organizacionais, que visa entender como o processo de cooperação proveniente das relações interorganizacionais influencia na cooperação das associações, neste estudo representadas pelas spin-offs analisadas (Astley, 1984; Astley \& Fombrun, 1983; Balestrin \& Verschoore Junior, 2010).

\subsection{Maturidade Tecnológica e Capacidade de Conversão de Conhecimento}

Mensurar a maturidade tecnológica e a valoração de tecnologias advindas do ambiente universitário, seja em spin-offs acadêmicas ou em outras empresas de base tecnológica, não é algo trivial. Apesar de existirem algumas formas apresentadas pela literatura para mensurar a valoração de tecnologias (Jiménez, 2015), e de investidores também possuírem suas metodologias próprias, muitas vezes baseadas no effectuation (Sarasvathy, 2001), dificilmente é possível presumir o sucesso de uma tecnologia ou produto, até porque estes dependem também de outros fatores, 
como membros fundadores (Diánez-González \& Camelo-Ordaz, 2016; Huynh et al., 2017), difusão de tecnologia (Rogers, 1983; Geroski, 2000), dentre outros.

No que diz respeito à maturidade tecnológica, este também é um processo complexo de mensuração e avaliação, visto que cada setor industrial possui suas peculiaridades (técnicas, de gestão, de mercado, etc.). Dentre algumas das escalas desenvolvidas para realizar a mensuração da maturidade tecnológica, recentemente a escala Technology Readiness Level (TRL) tem desempenhado papel importante para a mensuração do nível de desenvolvimento de produto e tecnologia em empresas de base tecnológica, principalmente na União Europeia, onde esta escala tem sido utilizada amplamente para mensurar projetos de inovação (Héder, 2017), bem como também tem sido adotada pelo Conselho Nacional de Ciência e Tecnologia (CONACYT) (Barron-Pastor \& García-Calderon, 2014), no México, que é o órgão governamental responsável pela promoção da ciência e tecnologia no país.

A maturidade tecnológica está relacionada diretamente com a capacidade de conversão de conhecimento da organização, visto que as duas estão relacionadas diretamente com o nível de desenvolvimento do produto ou tecnologia em empresas de base tecnológica. Desse modo, a capacidade de conversão do conhecimento é importante para o desenvolvimento de tecnologias e o avanço nos níveis de maturidade tecnológica.

Zahra, Van de Velde e Larra neta (2007), em seu artigo seminal sobre a aplicação da capacidade de conversão de conhecimento em spin-offs universitárias e spin-offs corporativas, dissertam sobre a diferença em que o constructo e suas dimensões são trabalhados nesses dois tipos de spin-offs. No trabalho, os autores colocam que a capacidade de conversão de conhecimento é dividida em três níveis: i) conceptualization and visioning capability; ii) configuration and design capability; iii) embodiment and integration capability.

O primeiro nível, conceptualization and visioning capability, é definido pelos autores como uma fase em que a equipe de gerentes analisa o conhecimento que possuem, explora e conceitua seus diferentes potenciais de aplicação, bem como determinam quem vai precisar dessas aplicações, e como estes possíveis clientes usarão os produtos e tecnologias oferecidos. Diz respeito à capacidade dos membros da spin-off de implantar recursos à medida que consideram diferentes usos e aplicações para suas tecnologias nascentes. Em suma, os autores definem como uma capacidade organizacional para explorar comercialmente o conhecimento das spin-offs.

O segundo nível, configuration and design capability, consiste, para os autores, no desenvolvimento de protótipos operacionais e funcionais que utilizam dessa tecnologia e configuram produtos que são possíveis de fabricar e comercializar. Assim, essa capacidade também inclui o desenvolvimento dos processos e sistemas que transformam produtos em produtos específicos.

Zahra, Van de Velde e Larraneta (2007) sustentam que o design de produtos feitos de uma forma efetiva influencia o custo, as estratégias de fornecimento, a facilidade de fabricação, o maquinário necessário e o treinamento recebido pelos empregados. Sousa-Ginel, Franco-Leal e Camelo-Ordaz (2017) colocam que a configuration and design capability está relacionada com a transferência de tecnologia, pois requer não apenas acesso à rede de conhecimento, mas também contempla todo o processo de transferência tecnológica, desde aquisição até a sua incorporação e aplicação dentro da spin-off acadêmica.

O terceiro nível, embodiment and integration capability, diz respeito à capacidade da empresa em transformar o conhecimento que possui em um produto ou serviço tecnológico aplicado. Segundo Zahra, Van de Velde e Larraneta (2007), as universidades e os centros de pesquisa são especializados em determinadas habilidades, que dizem respeito a peculiaridades da construção do conhecimento científico, ao aprofundamento e entendimento dos princípios de determinados fenômenos e tecnológicos - nas quais muitas vezes as aplicações práticas desses fenômenos e tecnologias não são trabalhadas o tanto quanto poderiam em suas mais diversas aplicações. Para Sousa-Ginel, Franco-Leal e Camelo-Ordaz (2017), este último nível está mais relacionado com a capacidade da organização de se beneficiar dos níveis anteriores, de modo a obter benefícios advindos da fase de pesquisa e transferência.

Fontes (2005) destaca o papel desempenhado das spin-offs acadêmicas de biotecnologia no processo de transformação do conhecimento científico e tecnológico. Em sua pesquisa, o autor cita as formas de intervenções das spin-offs acadêmicas no processo de transformação de conhecimento proveniente de organizações de pesquisas, bem como identifica e caracteriza três tipos de funções de transformação: i) as spin-offs que trazem ao mercado (direta ou indiretamente) o conhecimento produzido nas organizações de pesquisas na forma de tecnologias, produtos ou serviços; ii) as spin-offs que melhoram a acessibilidade ao conhecimento orientado para a indústria, sendo explorado por organizações de pesquisas, aumentando a qualidade do fornecimento e/ou ampliando a gama de usuários; e iii) as spin-offs que intermedeiam de forma ativa o conhecimento e/ou transferência de tecnologia de organizações de pesquisas e sua absorção por usuários específicos, com o intuito de identificar conhecimentos que possam responder a necessidades específicas.

Assim, seguindo os conceitos expostos por Zahra, Van de Velde e Larraneta (2007); Sousa-Ginel, Franco-Leal e Camelo-Ordaz (2017) é possível aferir que a capacidade de conversão do conhecimento é de extrema importância para as spin-offs acadêmicas. Ou seja, esta capacidade organizacional de converter determinado conhecimento em um produto ou tecnologia comerciável irá influir diretamente no seu desenvolvimento tecnológico, fazendo assim com que a empresa possa converter seu conhecimento em produtos ou tecnológias palpáveis. 


\section{PROCEDIMENTOS METODOLÓGICOS}

Para atender ao objetivo de compreender o papel das redes e da capacidade de conversão de conhecimento no desenvolvimento de spin-offs acadêmicas, foi desenhada e realizada uma pesquisa de campo de natureza qualitativa, dado o foco dessa perspectiva na compreensão dos fenômenos desde o ponto de vista dos atores envolvidos nos processos sociais, destacando-se a relação objetividade-subjetividade (Gaskell \& Bauer, 2002; Pires, 2008).

Quanto aos aspectos operacionais que envolvem o objetivo da pesquisa, a mesma se caracteriza como exploratório-descritiva, na medida em que explora um tema ainda pouco estudado pela literatura e o descreve num campo específico associado ao objeto de estudo. Quanto aos meios, foi utilizada a técnica de entrevistas individuais em profundidade como forma de coleta. Esta técnica objetiva a construção de informações pertinentes sobre o objeto, a partir da teoria que embasa a pesquisa realizada (Minayo, 2002).

Minayo (2002) aponta que a entrevista é um meio de representação de ideias, crenças, opiniões, sentimentos e comportamentos. Dessa forma, o método de entrevistas é oportuno para o tema estudado, visto que o objetivo é compreender e descrever os relacionamentos de spin-offs. Assim, pretendeu-se, por meio das entrevistas, conhecer para compreender a realidade dessas empresas por meio da expressão de seus gestores a respeito dos temas abordados.

Optou-se, portanto, por escolher diferentes spin-offs, atendendo ao princípio da diversificação na amostragem qualitativa (Pires, 2008), tendo em vista captar percepções do fenômeno, desde lugares específicos de vivência com o mesmo.

Os sujeitos entrevistados foram gestores e sócios das spin-offs, atuantes na área de biotecnologia, que possuíam conhecimento técnico sobre o desenvolvimento do produto, de forma que pudessem elaborar narrativas sobre o desenvolvimento da tecnologia e a transferência tecnológica do produto. Assim, foram entrevistados, no total, cinco profissionais a partir de uma análise prévia das empresas das quais faziam parte, de modo que os pesquisadores buscaram eleger empresas que de fato se classificavam como spin-offs acadêmicas.

Para que as empresas atendessem aos critérios, era necessario que fossem originadas a partir de conhecimento e pesquisa científica desenvolvido nas universidades de origem. Quanto aos gestores entrevistados, precisavam, necessariamente, ter participado da criação da empresa, e possuir conhecimento das áreas de gestão e tecnológia da empresa; bem como o deveria ser também pesquisador com conhecimento científico da área, o que foi o caso de todas as cinco empresas selecionadas.

Dessa forma, o quadro 1 apresenta o perfil dos cinco entrevistados:

Quadro 1-Perfil dos Entrevistados

\begin{tabular}{|c|l|l|l|l|l|l|l|}
\hline Sujeitos & $\begin{array}{c}\text { Campo da } \\
\text { Spin-off }\end{array}$ & \multicolumn{1}{|c|}{ Nível de maturidade } & $\begin{array}{c}\text { Tipo de tecnologia } \\
\text { do setor }\end{array}$ & Gênero & Idade & Escolaridade & \multicolumn{1}{|c|}{ Cargo } \\
\hline E1 & Biotecnologia & Comercialização tecnológica & Alta intensidade & Masculino & 34 & Doutorado & Sócio \\
\hline E2 & Biotecnologia & Aprimoramento da tecnologia & Alta intensidade & Masculino & 39 & Pós-doutorado & Gerente \\
\hline E3 & Biotecnologia & Comercialização tecnológica & Alta intensidade & Masculino & 35 & Pós-doutorado & Sócio \\
\hline E4 & Biotecnologia & Comercialização tecnológica & Baixa intensidade & Masculino & 36 & Pós-doutor & Fundador \\
\hline E5 & Biotecnologia & Comercialização tecnológica & Baixa intensidade & Masculino & 38 & Doutorado & Gerente \\
\hline
\end{tabular}

Fonte: elaborado pelos autores.

As entrevistas foram gravadas e as falas analisadas utilizando-se a técnica da análise temática de conteúdo, que, segundo Gomes (2013), possui os seguintes passos: categorização, inferência, descrição e interpretação. Segundo o autor, é preciso compreender o contexto em que está inserida a mensagem a ser analisada. Para isso, ele recomenda o uso de unidades de contexto como forma de categorização na análise de conteúdo temática.

Em seguida à categorização, é realizada a inferência sobre os dados por meio da constituição dos núcleos de sentido, que irão auxiliar na classificação das unidades de contexto em temáticas. Por fim, é realizada uma síntese interpretativa que dialogue os temas do campo com os objetivos, questões e pressupostos da pesquisa (Gomes, 2013).

Assim, esta pesquisa seguiu os passos sugeridos por Gomes (2013), visando a tornar o processo progressivamente exploratório, como: i) leitura flutuante das entrevistas; ii) leitura seletiva das entrevistas, com separação das unidades de contexto mais relevantes dentre as falas dos entrevistados; iii) identificação dos núcleos de sentido em cada unidade de contexto; iv) agrupamento dos núcleos de sentido convergentes; v) tematização dos blocos de núcleos agrupados; vi) validação dos temas, como testes de representatividade e consistência interna; vii) escrita interpretativa dos temas validados.

A partir da identificação dos núcleos de sentido convergentes e da classificação destas temáticas em blocos agrupados, foi possível separar os resultados coletados em quatro grandes grupos, posteriormente reduzidos em três para análise final. São eles: i) parcerias e redes de relacionamento; ii) capacidade de conversão de conhecimento; 
iii) desenvolvimento da spin-off; iv) aprendizado colaborativo. A temática de aprendizado colaborativo foi tratada nos resultados juntamente com a capacidade de conversão de conhecimento, pois ambas trataram sobre acumulação de conhecimento referente à tecnologia e aplicação deste conhecimento para fins comerciais.

Deste modo, foi possível delimitar a proporção entre o número de entrevistas que apresentaram núcleos de sentido ligados ao tema, sendo o número total de entrevistas denominado de representatividade; bem como a consistência interna formada pela quantidade de núcleos de sentido que um tema possui em relação a quantidade total dos núcleos de sentido.

Sendo assim, os temas que apresentaram menor representatividade e núcleos de sentido foram descartados ou agrupados com temas mais fortes, como subtemas para auxiliar no momento da explicação dos temas, facilitando a compreensão.

O Quadro 2 apresenta a análise da representatividade e da consistência interna dos temas.

Quadro 2 - Análise da representatividade e da consistência dos temas emergentes da categorização dos dados

\begin{tabular}{|l|c|c|}
\hline \multicolumn{1}{|c|}{ Temas } & Representatividade & Consistência interna \\
\hline Parcerias e redes de relacionamento & $5 / 5$ & 35 \\
\hline Capacidade de conversão de conhecimento & $5 / 5$ & 26 \\
\hline Desenvolvimento da spin-off & $5 / 5$ & 19 \\
\hline Aprendizado colaborativo & $4 / 5$ & 14 \\
\hline
\end{tabular}

Fonte: elaborado pelos autores.

Com o objetivo de atingir a saturação empírica, advinda da pesquisa de campo, que ocorre quando as últimas entrevistas realizadas não abordam informações novas (Pires, 2008), a pesquisa utiliza a abordagem de Fontanella et al. (2011), através da recorrência dos temas nas entrevistas, conforme quadro 3.

Quadro 3 - Recorrência dos temas nas entrevistas

\begin{tabular}{|l|c|c|c|c|c|c|}
\hline \multicolumn{1}{|c|}{ Temas } & E1 & E2 & E3 & E4 & E5 & Total de ocorrências \\
\hline Parcerias e redes de relacionamento & $!$ & + & + & + & + & 5 \\
\hline Capacidade de conversão de conhecimento & $!$ & + & + & + & + & 5 \\
\hline Desenvolvimento da spin-off & $!$ & + & + & + & + & 5 \\
\hline Aprendizado colaborativo & & $!$ & + & + & + & 4 \\
\hline Total de novos temas para cada entrevista & 3 & 1 & 0 & 0 & 0 & - \\
\hline
\end{tabular}

Legenda: ! = novo tema; + = recorrências.

Fonte: elaborado pelos autores, baseado em Fontanella et al. (2011).

\section{RESULTADOS}

A análise dos resultados apresenta os quatro temas emergentes da análise temática de conteúdo. Os quatro temas (parcerias e redes de relacionamento; aprendizado colaborativo; capacidade de conversão de conhecimento; desenvolvimento da spin-off) mostram-se convergentes com a literatura revisada na seção 2.

\subsection{Parcerias e Redes de Relacionamento}

As parcerias e as redes de relacionamento das spin-offs são importantes para o desenvolvimento das mesmas, principalmente no início das suas atividades (Huynh et al., 2017).

Isso é constatado na fala de E3 que considera a parceria com a universidade como fundamental, considerando que "ninguém faz nada sozinho". Este ainda considera que estas empresas devem buscar parcerias com organizações de maior porte que tem relevante influência no sistema de inovação regional, como as universidades e outras ICT's (Instituições de Ciência e Tecnologia). Desenvolver parcerias com empresas de múltiplos setores que a organização atua são primordiais para a empresa "acelerar a curva de crescimento daorganizacional" (Huynh et al., 2017).

É [...] a gente têm parceiros em todos os segmentos que a gente atua, tá? Na linha de biofármacos, que foi a que gente começou há mais tempo, é o segmento que temos mais parceiros. A gente tem parceiros hoje no Brasil, como fora do Brasil, se envolvendo cooperativamente, biofármacos. 
Então a gente não tá sozinho nessa. Na verdade não tem nenhum projeto de desenvolvimento de biofármacos que nós estejamos trabalhando sozinho. Todos têm parceiros. (E3).

A análise representada pelo relato acima corrobora com o paradigma da Teoria da Abordagem Estratégica de Redes, defendido por Astley (1984), Astley e Fombrun (1983), Balestrin e Verschoore Junior (2010), onde as estruturas em rede são eficientes para o alcance de objetivos organizacionais.

A capilaridade do relacionamento é nacional e internacional, viabilizada, conforme $\mathrm{E} 5$, pelo próprio relacionamento institucional da spin-off com a universidade de origem e pelos grupos de pesquisa. Os grupos de pesquisa, na visão de E5, permitem a divulgação e difusão da tecnologia no Brasil e no exterior e o aprimoramento da tecnologia com a troca de experiências e conhecimento.

Vários grupos de pesquisa foram nossos parceiros, como o do departamento de veterinária da universidade, os de biotecnologia que foram iniciados pelo professor $X$, inclusive um dos precursores da biotecnologia no estado. Tivemos também o departamento de física da universidade federal que veio em conjunto com a Universidade de Aveiro em Portugal e da Nova de Lisboa. (E5).

No que concerne à capilaridade de influência dos grupos de pesquisas no contexto da geração de inovações e tecnologias, o relato do entrevistado E5 demosntra diferentes níveis de colaboração dentro das redes, onde os membros estão inseridos em vários grupos de pesquisas, que pertencem a diferentes departamentos, vinculados em diversas universidades, reforçando o exposto na contribuição teórica de Brass (2004), onde a rede de cooperação interorganizacional compreende três níveis de composição: os membros, os grupos e as organizações.

Nas falas são destacadas a importância de parcerias para a comercialização do produto, atividade importante para uma spin-off (Walter, Auer, \& Ritter, 2006), que é indício de que a empresa atinge determinado nível de maturidade tecnológica.

Os gestores apontam para a importância da parceria com os profissionais dos setores nos quais atuam, pois estes possibilitam a comercialização e o desenvolvimento da tecnologia. No caso de E3 e E4, esses profissionais possibilitam a comercialização do produto em outros estados.

Na situação de E1, desenvolver um relacionamento com veterinários atuantes no campo é fundamental para acessar outra parceria importante para a empresa, que são pequenos produtores fornecedores de matéria-prima para o desenvolvimento da tecnologia.

A gente paga pelo serviço. [...] Então tem um serviço lá de fecundação in vitro. Aliás, temos as pessoas, os profissionais da região, que nós sabemos que pode dar o suporte pra aquilo que a gente não pode fazer. [...] Nós cobramos do cliente, e depois pagamos pelo serviço, de que a gente vai lá e cumpre o papel dele. (E3).

A gente tá aliando a parceria com o pequeno produtor para que a gente possa desenvolver o nosso produto. Não demandando custo de nossos projetos, seja projetos vinculados à universidade, seja de recurso próprio, da empresa, para despender na hora de contratar determinadas tecnologias, ou ainda produtos. Ou seja, a gente não gasta para utilizar. O pequeno produtor fornece o nosso principal material que a gente quer, que é o animal. [...] Sim, tá sendo uma parceria cativa, com esses produtores. E eu saliento, ainda, que um dos nossos principais parceiros são os médicos veterinários atuantes no campo. Porque hoje a gente tem uma técnica que a gente não oferece já para o produtor de forma direta. A gente trabalha em parceria com veterinários. No caso os veterinários fazem toda a parte de campo, e a gente toda a parte laboratorial. Isso que é interessante. [...] É, a gente chega no produtor através dos veterinários, né. Entra nessa parceria as universidades e as propriedades rurais. Mas o contato prévio são com os donos dessas instituições ou mesmo com os médicos veterinários. A porta de entrada é através deles. (E1).

Os relatos expostos acima fortalecem a corrente teórica que valida a importância das relações dos membros, dos grupos inseridos em associações e das associações que compõem as redes, visto que, essas relações estão alinhadas com interesses de desenvolvimento de produtos, transferência de tecnologia, compartilhamento de propriedade intelectual, além de aprendizado em mecanismos de gestão, o que não é visto na atuação de pesquisadores de forma isolada (Soetanto \& Van Geenhuizen, 2015; Huynh et al., 2017).

No caso de E4 devido a existência de um forte concorrente que coloca no mercado tecnologia semelhante, a empresa buscou realizar parcerias com os veterinários visando ganhar mercado ao invés de competir pelo consumidor final.

Como parei de vender para o cliente final, como o dono do animal, [...] do petshop, porque tínhamos dificuldades de ganhar mercado com este tipo de operação [...] passamos a trabalhar 
com o profissional do nicho que tem a competência para demandar nossos testes, indicar os testes para os clientes finais. (E4).

Uma das instituições parceiras que possui mais importância para as spin-offs são as universidades, afinal são elas que, muitas vezes, oferecem infraestrutura para as empresas nos seus primeiros anos. A fala de E1 ilustra bem a importância da mão de obra universitária para constituição das redes de relacionamento que contribuem para o desenvolvimento da empresa.

Alunos nossos, mestrandos, doutorandos, fazem a pesquisa aqui dentro conosco. É desenvolvido esse produto. Então com o desenvolvimento disso, a parceria. [...] Esse conjunto, esse contato mútuo entre universidade, que oferece como uma empresa incubada aqui dentro, oferece toda a estrutura física: equipamento, enfim. Que a gente acaba absorvendo essa mão de obra, que é altamente qualificada, de estudantes de mestrado e doutorado, a gente coloca aqui dento como alunos e estudantes, desenvolvendo pesquisa. E os sócios, que estão trabalhando a campo, captando clientes, captando esses parceiros, para que a gente possa desenvolver e financiar de alguma forma essas pesquisas. É assim que funciona. O ciclo é dessa maneira. (E1).

Além disso, E1, E4 e E5 utilizam o capital intelectual da universidade para desenvolver suas pesquisas tecnológicas e desenvolver seus produtos. No caso de E4 a formação acadêmica de doutorado e pós-doutorado do gestor da corporação foi na universidade na qual é incubada, bem como a de seu sócio.

Já no caso de E5 toda a infraestrutura da universidade apoiou no desenvolvimento da tecnologia dentro das possibilidades de cooperação da mesma. Conforme o entrevistado, a universidade só não colaborou mais por causa da própria disponibilidade de cursos e campos de engenharia, o que comprometeu, de certa maneira, a performance evolutiva da empresa, bem como também impediu a organização a buscar parcerias com outras universidades, grupos de pesquisa e parceiros de testes, sobretudo para validação das tecnologias em todas as etapas do desenvolvimento do produto.

As universidades também possibilitam outras oportunidades para o desenvolvimento das spin-offs, como financiamento, suporte de infraestrutura, incubação e mentoria (Soetanto \& Van Geenhuizen, 2015; Wright, Siegel, \& Mustar, 2017).

Nos casos de E2 e E4 a incubadora da universidade foi primordial na oferta de cursos e treinamentos, o que possibilitou a remodelação do produto para que este tivesse uma maior inclinação e aceitação no mercado, uma vez que os pesquisadores têm uma formação técnica e pouco gerencial nas spin-offs acadêmicas pesquisadas. Assim, as oportunidades oferecidas pela universidade são também percebidas por E1 e E3, por exemplo, no caso daquele como um valor agregado.

Eu ressalto que hoje é uma vantagem muito grande estar dentro da universidade 1. É um valor agregado muito grande na nossa estrutura, que não saiu do nosso bolso. E sim a universidade 1 que ofereceu a oportunidade da gente, como empresa incubada, passar por um período de amadurecimento aqui dentro. A gente desenvolveu os nossos produtos pra chegar numa graduação da nossa empresa eficiente, para que a gente possa andar com as nossas próprias pernas. (E1).

[...] E a universidade 2, além de ter abrigado esse projeto, ter gostado, aberto as portas, ela também passou [...] ela acreditou e passou a investir no projeto. Mas então há um financiamento permanente da universidade fomentando esse segmento, tá? Além disso, nós incubamos a empresa aqui dentro. A empresa rodando com baixo custo de manutenção. A universidade 2 gostou da parceria com a empresa. Poderia haver, de repente, um conflito de interesses, né. Mas não houve. Na verdade, a universidade tá muito bem. Tá muito bem alinhada com esse tipo de iniciativa, e preocupada com essa conexão com o mercado. E entende a importância das startups, empresas em geral. [...] Então, além dela investir, dela abrir as portas como uma incubadora de empresas, ela também abriu, recentemente, um parque tecnológico, e ela vive trabalhando pra fazer as iniciativas darem certo. [...] E a universidade 2 tá sempre fomentando esse negócio, fazendo com que aconteça, se preocupando com as conexões, trazendo as pessoas de fora. [...] Então a universidade 2, é... a universidade, no nosso caso, tem sido fantástica. Sei que é uma exceção. (E3). 


\subsection{Aprendizado Colaborativo e Capacidade de Conversão de Conhecimento}

O aprendizado colaborativo está diretamente relacionado com capacidade de conversão de conhecimento, pois trata sobre a acumulação do conhecimento referente a uma determinada tecnologia onde esse conhecimento adquirido volta para a empresa por meio do compartilhamento do aprendizado no desenvolvimento de novas tecnologias. A fala de E3 ilustra bem essa relação:

A gente precisa de um volume muito grande de pessoas para desenvolver as tecnologias. [...] Então eu preciso de parceiros, que são os alunos, também, que funcionam como a nossa maõ de obra mas que detêm também hoje a tecnologia que a gente desenvolve aqui dentro. De eficiência na parte de aquisição de conhecimento, hoje os alunos trabalham com as tecnologias de ponta, altamente aplicáveis, tanto no campo quanto no laboratório. Então isso aí já traz um benefício tremendo para esses alunos. Ou seja, nós estamos desenvolvendo tecnologia que está sendo aplicada a partir de alunos que já vem em uma linha de pesquisa, em uma linha de atuação já há bastante tempo. Focados nas pesquisas que a gente desenvolve. (E3).

Percebe-se, na fala acima, que o trabalho desenvolvido pelos alunos gera um aprendizado tanto para eles próprios quanto para a empresa, o que mostra a importância do aprendizado colaborativo como da capacidade de conversão de conhecimento.

A fala de E2, abaixo, também reforça a relação entre aprendizado colaborativo e a conversão do conhecimento mostrando o benefício do aprendizado para o desenvolvimento da tecnologia aliado às parcerias.

Nós precisamos que alguns dos nossos pesquisadores interajam com os pesquisadores de outros grupos para poder fazer a funcionalização desses equipamentos com o produto, né? Então o aprendizado sempre existe, e também quando você faz uma parceria com outras empresas, você sempre está discutindo algumas formas de gestão, de gestão de projetos, então sempre um aprendizado que é analisado de uma coisa para outra. Então sempre existe um aprendizado, ou uma nova tecnologia que está disponível no mercado. Ou em pesquisas mesmo, e geralmente você colabora com esses outros parceiros. [...] Com esses competidores, parceiros, através de reuniões, ou de congressos, onde você discute como tá sendo desenvolvido toda a tecnologia, né? (E2).

Quanto à capacidade de conversão do conhecimento, nas spin-offs acadêmicas existe a ampliação destes processos, como destacam Rogers (1983) e Geroski (2000) quando os cientistas expõem seus produtos tecnológicos em situações de competição e de avaliação dos pares.

Todo o setor é muito dinâmico, né? Então sempre existe um aprendizado, ou uma nova tecnologia que está disponível no mercado, ou em pesquisas mesmo, e geralmente você colabora com esses outros parceiros [...]. Com esses competidores, parceiros, através de reuniões, ou de congressos, onde você discute como tá sendo desenvolvido toda a tecnologia, né? Principalmente porque a gente trabalha com a tecnologia que é muito de ponta mesmo. Você tem que tá sempre se adaptando e melhorando pra poder fazer realmente isso aí funcionar. (E2).

Estas atividades não só ampliam o conhecimento das equipes e gerentes de spin-offs acadêmicas, mas também despertam as possibilidades de novas aplicações do produto tecnológico e auxiliam na exploração comercial tecnológica, como descrito na conceptualization and visioning capability de Zahra, Van de Velde e Larraneta (2007).

Para E4 demonstrar o produto tecnológico é uma experiência que abre portas para sua comercialização do produto, como na possibilidade de prestar o serviço tecnológico para novos clientes pesquisadores ou pessoas indicadas pelos demais cientistas. O entrevistado destaca "lá na frente nos ajuda a aprimorar atividades administrativas e a alterar rotinas para prestação do serviço de diagnóstico".

Na compreensão de Sousa-Ginel, Franco-Leal e Camelo-Ordaz (2017) o processo de conversão de conhecimento está intimamente ligado à rede e também de todo o processo de transferência tecnológico.

Para E5 a falta de recursos financeiros provoca a necessidade de compartilhar parte das etapas de desenvolvimento tecnológico, mas existe uma relação de "ganha-ganha" com os parceiros, que para E2 não pode ser qualquer um e deve passar por uma triagem para que o processo ocorra.

[...] O que seria de benefício: primeiro, pesquisadores parceiros que trabalham conosco dentro, in loco, dentro do nosso laboratório, trazem uma tecnologia pra nós, tá? [...] Tem que ter um contato prévio e uma confiança muito grande, que muitos produtos aqui são segredos industriais. (E2). 
Já para de $\mathrm{E} 3$, a rede de colaboração acaba melhorando não só o desenvolvimento da tecnologia como a performance da organização em outros setores quando usufrui do ambiente, no qual as spin-offs acadêmicas estão imersas, como outros pesquisadores, grupos de pesquisa, profissionais do mercado, usuários da tecnologia e empresas parceiras.

[...] Talvez se a gente tivesse simplesmente a empresa, talvez fosse mais fechado na questão financeira/comercial, talvez a gente não tivesse isso tão na mão. Mas como a gente consegue conectar o que é feito na empresa, a transferência de tecnologia com ciência, com pesquisa, a gente acaba conseguindo usufruir desse ambiente, e dessas pessoas, desses outros pesquisadores. (E3).

Com relação à fala de E3, é interessante notar quais são os benefícios ao colaborar com outras empresas de acordo com o nível de maturidade da tecnologia. Quando o produto ainda está em fase de transferência de tecnologia para o mercado, o interesse está na obtenção de know-how e na captação de recursos necessários para o desenvolvimento da tecnologia.

Já quando o produto está em um nível de maturidade tecnológica para ser comercializado, o maior interesse é em colaborar com outras empresas para possibilitar a venda do produto. No caso de E5, como o produto já estava bem desenvolvido, a possibilidade de parceria com outras empresas servia para solucionar problemas da spin-off e da empresa parceira, corroborando para o desenvolvimento de tecnologias desenvolvidas em conjunto. No entanto, dificuldades foram encontradas com relação a E4 e a venda da tecnologia.

Tivemos resultados no desenvolvimento de produtos, mas esses resultados trouxeram questões difíceis de lidar como com relação à venda da tecnologia [...], como desenvolvemos a tecnologia juntos não conseguimos chegar a um consenso para comercializar a tecnologia, por causa de questões burocráticas com relação ao registro da propriedade intelectual. (E4).

Sousa-Ginel, Franco-Leal e Camelo-Ordaz (2017) propõem o desenho da tecnologia e suas configurações como uma importante ferramenta para o processo de desenvolvimento tecnológico nas organizações com a realização de reuniões e ciclos de controle de qualidade. E5 destaca que os problemas são as atividades que auxiliam na busca por não conformidades do produto. Em E2 é mantido um processo de fluxo contínuo para solução de problemas e em E4 o processo é utilizado até como forma de aprendizado, bem como na adequação e reformulação dos protocolos usados nos testes com a tecnologia.

\subsection{Desenvolvimento da Spin-Off}

As spin-offs precisam superar desafios durante a sua fase de criação e desenvolvimento, comum a toda empresa iniciante. Esse tipo de empresa também precisa se preocupar, em muitos casos, como a transferência de tecnologia do seu produto (Djokovic \& Souitaris, 2008; Freitas et al., 2011). Sendo assim, a empresa precisa desenvolver soluções para que possa sobreviver durante seu período inicial. A fala de E1 cita como a empresa desse gestor busca superar essasbarreiras.

Hoje em dia, para reduzir a nossa folha de pagamento, digamos assim, quem trabalham são somente os sócios, tá? Como funcionário, digamos assim, nós temos hoje quatro sócios, né? A gente aproveita a mão de obra da universidade. Como a gente trabalha em parceria com os laboratórios a gente aproveita alunos de iniciação científica, mestrandos, doutorandos, ou até mesmo pós-docs. Ou seja, mão de obra altamente qualificada e de baixo custo pra gente. Isso é interessante. Esse vínculo com as instituições públicas permite que alunos, com bolsas prévias nas mais diferentes instituições, trabalhem desenvolvendo a nossa tecnologia aqui dentro, na qual a gente pode oferecer futuramente. (E1).

Para superar dificuldades inicias, as spin-offs recorrem a parcerias, procuram opções de financiamento (Pérez \& Sánchez, 2003; Soetanto \& Van Geenhuizen, 2015; Wright, Siegel, \& Mustar, 2017) e know-how para o desenvolvimento das suas tecnologias.

A empresa E4 conseguiu se desenvolver com a captação de recursos por meio de um edital de projeto de financiamento da universidade, que permitiu que a organização tivesse condições de equipar melhor o laboratório e comprar material de consumo para realizar experiências que viabilizaram o desenvolvimento tecnológico. Em sua fala, E3 coloca que viu a necessidade da criação de uma empresa para superar os desafios comuns às spin-offs acadêmicas. 
E nós vimos essa possibilidade de abrir uma empresa como um braço a mais pra captação de recurso adicional, de parceiro adicional. A parceria com uma empresa, universidade-empresa, ela morre antes mesmo de começar, pela simples demora das duas partes entregarem o termo de sigilo assinado, depois um plano de trabalho assinado. [...] Logo, no meio isso aconteceu algumas vezes. E quando você consegue fazer isso com empresa, você acelera tudo, fica mais simples. Bom, esse foi o intuito inicial da empresa. Construir essa ponte entre uma pesquisa da universidade 2. Então nós incubamos a empresa aqui dentro, e começamos, a partir de 2014, a captar recursos adicionais em editais que nós não teríamos acesso. E fizemos parcerias com empresas, e tá dando certo o modelo. (E3).

\subsection{Discussão dos Resultados}

As redes de colaboração e compartilhamento são importantes para a inovação e a transferência de tecnologia no contexto universitário. Dentro desse contexto, existem vários mecanismos de transferência de tecnologia da universidade para o setor produtivo, sendo um dos mais comuns a criação de spin-offs acadêmicas (Etzkowitz, 1998; Shane, 2004; Ferraro \& lovanella, 2017). Nesse ambiente universitário, de produção, troca e transferência de conhecimento, as redes se apresentam como alternativa para estratégias de cooperação entre as organizações que atuam no papel de transferência tecnológica e indivíduos que possuem o objetivo de produzir inovação e viabilizar a transferência de tecnologias produzidas no ambiente acadêmico (Powell \& Koput; Smith-Doerr, 1996; Tsai, 2001; Balestrin \& Verschoore, 2010; Ferraro \&lovanella, 2017).

Balestrin e Verschoore (2010) demonstram, por exemplo, como a transferência de conhecimento através da colaboração em rede faz com que empresas possam adotar novas práticas de trabalho e produzir novos produtos, gerando aprendizado e inovação. Neste sentido, as spin-offs acadêmicas se estabelecem como instituição com o papel de transferir a tecnologia produzida no ambiente universitário e, desta forma, agregar valor econômico, gerando riquezas para a sociedade (Etzkowitz, 1998; Shane, 2004).

Durante esta pesquisa, constatou-se que as redes de relacionamento das spin-offs acadêmicas contribuem para a formação de parcerias que usam a colaboração, mesmo num ambiente de competição, como meio para atingir objetivos comuns (BALESTRIN; VERSCHOORE, 2010). Foi possível, então, notar que as redes se constituíam não apenas para fins de benchmarking (comparação de produtos, serviços e práticas comerciais) e aquisição de novas tecnologias, mas sim que eram formadas parcerias com diferentes atores, com o objetivo que a empresa pudesse aprender em diferentes áreas, gerando, a partir disto, vantagens competitivas (Perez \& Sánchez, 2003; Walter, Auer, \& Ritter, 2006), bem como construindo capacidades para o seu estabelecimento no mercado e desenvolvimento organizacional (Boccardelli \& Magnusson, 2006).

Isto diz respeito tanto na área técnica e tecnológica da empresa, com relação à assimilação de conhecimento nesta área a partir de outra empresa ou de um laboratório da área, quanto na área de gestão, com troca de conhecimento nas áreas comerciais, tratando sobre os processos de entrada no mercado e das etapas da comercialização da tecnologia. Assim, foi possível notar que estes relacionamentos são estabelecidos com base na confiança mútua entre as partes (Tsai, 2001; Capaldo, 2007), com um longo período de tempo para o amadurecimento destas relações.

Deste modo, a partir do estabelecimento das relações entre empresa e diferentes atores (universidades; laboratórios; empresas parceiras e concorrentes; clientes; pesquisadores), é gerado um acúmulo de conhecimento na área de atuação e na gestão da spin-off (Diánez-González \& Camelo-Ordaz, 2016; Huynh et al., 2017). Este aprendizado e acúmulo de conhecimento se refletem em um importante construto para o desenvolvimento tecnológico da empresa, que é a capacidade de conversão de conhecimento (Walter, Auer, \& Ritter, 2006; Sousa-Ginel, Franco-Leal, \& Camelo-Ordaz).

O desenvolvimento da tecnologia da empresa, aprimorando a parte técnica e a sua aplicação para fins comerciais, é fundamental para o desenvolvimento da empresa e para o seu desempenho (Birkinshaw \& Mol, 2008; Damanpour, Walker, \& Avellaneda, 2009; Jiménez-Jiménez \& Sanz-Valle, 2011). Assim, estes achados contribuem com a literatura no sentido de apontar como ocorrem a relação entre estes diferentes construtos, com ênfase na necessidade da confiança e da colaboração, como apontam Tsai (2001) e Capaldo (2007), como necessários para que, de fato, as redes possam impactar nos demais construtos.

Neste sentido, a pesquisa corrobora com outros estudos da área (Diánez-González \& Camelo-Ordaz, 2016; Huynh et al., 2017), e apresenta indícios de que os níveis de parcerias e colaboração irão moderar os benefícios gerados a partir da inserção da empresa nas redes, apontando que índices mais altos de colaboração irão refletir em maior aprendizado, maior capacidade de conversão de conhecimento e, consequentemente, no desenvolvimento da spin-off. 


\section{CONSIDERAÇÕES FINAIS}

A partir do estudo exploratório realizado, com base nos dados coletados e promoção, pode-se afirmar que o objetivo desta pesquisa foi alcançado pelo processo de compreensão do papel das redes de cooperação e da capacidade de conversão de conhecimento no desenvolvimento de spin- offs acadêmicas.

As redes de cooperação, analisadas nesta pesquisa, demonstraram atuar como um mecanismo de vantagem competitiva para as spin-offs acadêmicas, que utilizam um ambiente de cooperação para desenvolver o aprendizado colaborativo com o objetivo de potencializar a capacidade de conversão do conhecimento em tecnologias comercializáveis.

Neste ensejo, constatou-se, também, que as redes de relacionamento das spin-offs acadêmicas criam vínculos colaborativos para atingir os objetivos em comum, reunindo indivíduos e grupos que possuem diferentes competências técnicas e gerenciais.

Algumas das identificadas, neste estudo, é a otimização da utilização dos recursos materiais, muitas vezes escassos, no processo de criação de inovações e produção de tecnologias para o mercado. Além disso, as relações de trocas entre os indíviduos inseridos em diferentes grupos da rede são pautadas na confiança e no amadurecimento temporal, onde a maturidade dessas relações é influenciada pela recorrência de trocas e aprendizagens mútuas.

No que diz respeito ao aprendizado colaborativo e à capacidade de conversão do conhecimento, o estudo realizado permite que a construção de aprendizagem e o acúmulo de conhecimento entre os individuos dessas redes representam construtos importantes para o desenvolvimento tecnológico das spin-offs. O crescimento do potencial tecnológico dessas empresas reflete no aprimoramento de suas áreas técnicas e comerciais, o que influencia de forma positiva na maturidade e desempenho das spin-offs no mercado.

Quanto às limitações do estudo, pode-se apontar a quantidade de gestores entrevistados, que se limitaram a cinco membros de diferentes spin-offs. Assim, o número reduzido limita os resultados finais da pesquisa, o que requer ampliação do escopo para aparofundamento das análises.

Como recomendações de estudos futuros, sugere-se pesquisas que tratem dos mesmos temas em spin-offs de outros setores, além do setor de biotecnologia; coleta de dados com membros que possuem outros cargos nas empresas, além de cargos de gestão, e com parceiros das spin-offs, de forma a possibilitar análises sob diferentes perspectivas no que diz respeito às informações dessas empresas; e, por fim, a aplicação de pesquisas quantitativas com os temas tratados neste estudo, de modo a testar as hipóteses das relações entre os construtos abordados nesta pesquisa.

\section{REFERÊNCIAS}

Balestrin, A., \& Verschoore, J. (2010). Aprendizagem e inovação no contexto das redes de cooperação entre pequenas e médias empresas. Organizações \& Sociedade, 17(53).

Barron-Pastor, D., \& García-Calderon, N. (2014). Conceptos básicos de Gestión de Tecnología y Propiedad Intelectual para investigadores y emprendedores científico tecnológicos. Centro de Investigación Científica de Yucatán.

Bauer, M. W., \& Gaskell, G. (2017). Pesquisa qualitativa com texto, imagem e som: um manual prático. Editora VozesLimitada.

Birkinshaw, J., Hamel, G., \& Mol, M. J. (2008). Management innovation. Academy of management Review, 33(4), 825-845.

Boaventura, J. M. G., Carnaúba, A. A. C., Todeva, E., Azevedo, A. C., \& Armando, E. (2016). Governance structures and trust: A study of real estate networks. Journal on Chain and Network Science, 16(2), 157-170.

Boccardelli, P., \& Magnusson, M. G. (2006). Dynamic capabilities in early-phase entrepreneurship. Knowledge and Process Management, 13(3), 162-174.

Breschi, S., \& Malerba, F. (2005). Clusters, networks and innovation. Oxford University Press.

Capaldo, A. (2007). Network structure and innovation: The leveraging of a dual network as a distinctive relational capability. Strategic management journal, 28(6), 585-608.

Clarysse, B., \& Moray, N. (2004). A process study of entrepreneurial team formation: The case of a research-based spin-off. Journal of Business Venturing, 19(1), 55-79.

Cullen, J. B., Johnson, J. L., \& Sakano, T. (2000). Success through commitment and trust: The soft side of strategic 
alliance management. Journal of World Business, 35(3), 223-240.

Damanpour, F., Walker, R. M., \& Avellaneda, C. N. (2009). Combinative effects of innovation types and organizational performance: A longitudinal study of service organizations. Journal of management studies, 46(4), 650-675.

Diánez-González, J. P., \& Camelo-Ordaz, C. (2016). How management team composition affects academic spin-offs' entrepreneurial orientation: The mediating role of conflict. The Journal of Technology Transfer, 41(3), 530-557.

Djokovic, D., \& Souitaris, V. (2008). Spinouts from academic institutions: a literature review with suggestions for further research. The Journal of Technology Transfer, 33(3), 225-247.

Etzkowitz, H. (1998). The norms of entrepreneurial science: Cognitive effects of the new university-industry linkages. Research policy, 27(8), 823-833.

Ferraro, G., \& lovanella, A. (2017). Technology transfer in innovation networks: An empirical study of the Enterprise Europe Network. International Journal of Engineering Business Management, 9, 1-14.

Fontanella, B. J. B., Luchesi, B. M., Saidel, M. G. B., Ricas, J., Turato, E. R., \& Melo, D. G. (2011). Amostragem em pesquisas qualitativas: proposta de procedimentos para constatar saturação teórica. Cadernos de Saúde Pública, 27, 388-394.

Fontes, M. (2005). The process of transformation of scientific and technological knowledge into economic value conducted by biotechnology spin-offs. Technovation,25(4), 339-347.

Freitas, J. S., Gonçalves, C. A., Cheng, L. C., \& Muniz, R. M. (2011). O fenômeno das spin-offs acadêmicas: Estruturando um novo campo de pesquisa no Brasil. RAI Revista de Administração e Inovação, 8(4), 67-87.

Frota, R. A., \& Freitas, A. A. F. (2018). Comunicação em Spin-Offsacadêmicas: Um estudo exploratório em empresas de base biotecnológica. Revista de Empreendedorismo e Gestão de Pequenas Empresas, 7(1),71-103.

Funk, R. J. (2014). Making the most of where you are: Geography, networks, and innovation in organizations. Academy of Management Journal, 57(1), 193-222.

Geroski, P. A. (2000). Models of technology diffusion. Research policy, 29(4-5), 603-625.

Gomes, R. (2013). Análise e interpretação de dados de pesquisa qualitativa. In M. C. Minayo, S. F. Deslandes \& R. Gomes, Pesquisa social teoria, método e criatividade (33 ed.).Vozes.

Huynh, T., Patton, D., Arias-Aranda, D., \& Molina-Fernández, L. M. (2017). University spin- off's performance: Capabilities and networks of founding teams at creation phase. Journal of Business Research, 78, 10-22.

Ipiranga, A. S. R., \& Almeida, P. C. H. (2012). O tipo de pesquisa e a cooperação universidade, empresa e governo: Uma análise na rede nordeste de biotecnologia. Organizações \& Sociedade, 19(60).

Jiménez Hernández, C. N. (2015).Propuesta para la valoración tecnológica desde la perspectiva de la síntesis evolutiva moderna. Doctoral dissertation, Universidad Nacional de Colombia, Bogotá.

Jiménez-Jiménez, D., \& Sanz-Valle, R. (2011). Innovation, organizational learning, and performance. Journal of business research, 64(4), 408-417.

Minayo, M. C. (2002). Pesquisa social: teoria e método. Vozes.

Mosey, S., \& Wright, M. (2007). From human capital to social capital: A longitudinal study of technology-based academic entrepreneurs. Entrepreneurship theory and practice, 31(6), 909- 935.

O'Shea, R. P., Chugh, H., \& Allen, T. J. (2008). Determinants and consequences of university spinoff activity: a conceptual framework. The Journal of Technology Transfer, 33(6), 653-666.

Perez, M. P., \& Sánchez, A. M. (2003). The development of university spin-offs: Early dynamics of technology transfer 
and networking. Technovation, 23(10),823-831.

Pires, Á. P. (2008). Amostragem e pesquisa qualitativa: Ensaio teórico e metodológico. In J. Poupart, J. P. Deslauriers, L. H. Groulx, A. Lapemère, R. Mayer, \& A. P. Pires (Org.), A pesquisa qualitativa: enfoques epistemológicos e metodológicos. Vozes.p. 154-211.

Powell, W. W., Koput, K. W., \& Smith-Doerr, L. (1996). Interorganizational collaboration and the locus of innovation: Networks of learning in biotechnology. Administrative science quarterly, 41(1),116-145.

Rogers, E. M., \& Williams, D. (1983). Diffusion ofInnovations. The Free Press.

Sarasvathy, S. D. (2001). Causation and effectuation: Toward a theoretical shift from economic inevitability to entrepreneurial contingency. Academy of management Review, 26(2), 243-263.

Soetanto, D., \& Van Geenhuizen, M. (2015). Getting the right balance: University networks' influence on spin-offs' attraction of funding for innovation. Technovation, 36, 26-38.

Sousa-Ginel, E., Franco-Leal, N., \& Camelo-Ordaz, C. (2017). The influence of networks on the knowledge conversion capability of academic spin-offs. Industrial and Corporate Change, 26(6), 1125-1144.

Tsai, W. (2001). Knowledge transfer in intraorganizational networks: Effects of network position and absorptive capacity on business unit innovation and performance. Academy of management journal, 44(5), 996-1004.

Walter, A., Auer, M., \& Ritter, T. (2006). The impact of network capabilities and entrepreneurial orientation on university spin-off performance. Journal of business venturing, 21(4), 541-567.

Wright, M., Siegel, D. S., \& Mustar, P. (2017). An emerging ecosystem for student start-ups. The Journal of Technology Transfer, 42(4), 909-922.

Zaheer, A., McEvily, B., \& Perrone, V. (1998). Does trust matter? Exploring the effects of interorganizational and interpersonal trust on performance. Organization science, 9(2), 141-159.

Zahra, S. A., Van de Velde, E., \& Larraneta, B. (2007). Knowledge conversion capability and the performance of corporate and university spin-offs. Industrial and Corporate Change, 16(4), 569-608.

\section{Contato:}

Brenno Buarque

E-mail: brenno_buarque@hotmail.com

Ana Cristina Batista dos Santos

E-mail: ana.batista@uece.br

Naiderson Ferreira de Lucena

E-mail: naidersonlucena@gmail.com

Rafaela Cajado Magalhães

E-mail: rafaela.cajado0105@gmail.com

Herus Orsano Machado

E-mail: herus.machado@ifma.edu.br 